\title{
El movimiento de ecoaldeas como experiencia alternativa de Buen Vivir
}

\author{
Leonardo Salamanca López \\ Corporación Universitaria Minuto de Dios (Uniminuto), Bogotá, Colombia. \\ Email: hleontes@gmail.com \\ Diego Fernando Silva Prada \\ Corporación Universitaria Minuto de Dios (Unimiuto) Bogotá, Colombia. \\ Email: silvaprada@yahoo.es
}

\begin{abstract}
Resumen: Este artículo pretende mostrar un acercamiento histórico y teórico conceptual al fenómeno social de las ecoaldeas, empezando por la descripción de su emergencia en el siglo XX para luegoseñalar la envergadura global del movimiento desde la construcción de redes regionales en todo el mundo. Enseguida se desarrollarán dos aspectos del movimiento de ecoaldeas como objeto de estudio social para luego trabajar tres principios fundamentales de su acción. Se realizará una evaluación de las problemáticas desde las que surgen las ecoaldeas y las respuestas generadas por ellas en cuanto que comunidades intencionales que concretizanel Buen Vivir desde los procesos comunitarios de neoruralidad. Se pretende responder a la cuestión de en qué medida las ecoaldeas son o no una alternativa real al sistema capitalista fundamentadas en la idea del Buen Vivir.
\end{abstract}

Palabras clave: Ecoaldeas, Buen vivir, desfetichización, comunidad, alternativas al capitalismo.

\section{The ecovillage movement as an alternative experience of Good Living}

\begin{abstract}
This article aims to show an historical and theoretical conceptual approach to the social phenomena of ecovillages, starting with the description of its emergence in the twentieth century, and then point to the overall size of the movement from the construction of regional networks around the world. Then two aspects of the ecovillage movement as an object of social study and three fundamental principles of action will be developed. An assessment of the problems from which ecovillages arise and responses generated by them as intentional communities that concretize Good Living arising from neoruralitycommunityprocesses. Weattempt to answer the question of to what extent ecovillages are or aren't a real alternative to capitalist system founded on the idea of ??Good Living. to capitalism.

Keywords: Ecovillage, Good living, defetishisation, community, alternatives
\end{abstract}

\section{O movimento das ecovilas} como uma experiência alternativa de Bom Viver

Resumo: Este artigo tem como objetivo mostrar uma abordagem conceitual 
histórica e teórica para ecovillages fenômenos sociais, a partir da descrição de seu surgimento no século XX e, em seguida, apontar a partir da construção de redes regionais em todo o mundo para o tamanho total do movimento. Em seguida, dois aspectos do movimento das ecovilas como um objeto de estudo social e, em seguida, trabalhar três princípios fundamentais da acção será desenvolvida. Uma avaliação dos problemas decorrentes de ecovilas e respostas geradas por eles em que as comunidades intencionais que concretizam boa vida de processos comunitários neoruralidad são executadas. Destina-se a responder à pergunta o que ecovillages extensão ou não um sistema capitalista real, fundada na idéia de Boa alternativa Vivo.

Palavras-chave: Ecovillage, Lar de Idosos, defetishisation, alternativas comunitárias ao capitalismo.

\section{Introducción: orígenes de las ecoaldeas como fenómeno social ${ }^{1}$}

Podemos comenzar por afirmar la existencia de un doble carácter respecto al origen histórico y social de las ecoaldeas: son un fenómeno antiguo y nuevo a la vez. Como tal, las ecoaldeas tienen un antecedente directo en lo que se conoce como los Kibbutz judíos o comunas agrícolas israelíes que comenzaron en el año de 1910 a partir de migrantes rusos con una fuerte influencia socialista (Montoya \& Dávila 2005: 36); basado en ideales de ser un poblado rural "multigeneracional, caracterizado por la vida en comunidad, por su administración democrática, responsabilidad por el bienestar de cada uno de sus miembros, niños y adultos, por la salud y la educación, y por la copropiedad de los medios de producción y de consumo” (Organización Kibbutz 2014). Igualmente, otro antecedente directo se halla en la experiencia del movimiento hippie de los años sesenta norteamericano, en donde la contracultura como crítica al materialismo reinante, la libertad sexualy la experiencia comunitaria fueron los pilares de una experiencia de ruptura frente al sistema capitalista y de construcción de alternativas sociales desde la solidaridad, el naturalismo, el arte y el amor (De los Ríos 1998). En la bibliografía disponible sobre este fenómeno encontramos otras amplias líneas de posibles influencias como las experiencias de las comunidades Ashrams en la India, los Lamas en el Tíbet, los Amishes en Estados Unidos y los Ayllus de los Ándes (Barney 2011), los Calpullis aztecas, koljoz y sovjos y cuáqueros (Ruz 2014: 26).

Desde el lado novedoso, encontramos que el término es utilizado sistemáticamente por George Ramsey en la Primera Conferencia Mundial de Energía para 1978. Aunque para la misma época, la revista Noticias de la Madre Tierra en Estados Unidos ya hablaba de “eco-aldeas” para denominar los sistemas de energía experimental y los jardines orgánicos cerca de oficinas en Hendersonville, en Carolina del Norte (Bates 2003). Así mismo, el fenómeno tuvo paralelismo en Alemania en el contexto de la lucha antinuclear, al construirse aldeas pequeñas y ecológicas en la localidad de Gorleben, en alemán ökodorf (ecoaldea) como forma de resistencia a la 
generación de energía nuclear y sus consecuencias negativas ya sus peligros. Sin embargo, como iniciativa y práctica concreta, es Findhorn en Escocia el momento inaugural de las ecoaldeas, al ser emprendida esta nueva experiencia en 1962 por Peter y Eileen Caddyjunto con Dorothy Maclean y luego de diez años constituirse legamente como Fundación Findhorm (Findhorn Fundation, 2014) ${ }^{2}$.

Para 1991 el fenómeno se ha generalizado y profundizado, siendo teorizado por Robert Gilman, mostrando a las comunidades que responden a los desafíos de la vida urbana moderna. Así, una ecoaldea es definida como:

un asentamiento humano e integral (no solo es una estructura de viviendas, sino un asentamiento donde las actividades humanas están integradas en el medio natural de manera inocua), concebido a escala humana, que incluye todos los aspectos importantes para la vida, integrándolos respetuosamente en el entorno natural, que apoya formas saludables de desarrollo (sostenible/sustentable) y que pueda persistir indefinidamente (1995: 19).

Así mismo podemos encontrar esta definición histórica:

En los años setenta grupos de personas empezaron a crear comunidades en las que uno de los objetivos centrales era volver al campo proponiendo una vida en comunidad, produciendo y consumiendo a nivel local y cerca de la naturaleza. Las comunidades intencionales, diferentes a las comunidades que por tradición no han elegido su lugar en el mundo, van en contra de la tendencia actual de ir a la ciudad ?motivados principalmente? por la crisis social, la degradación ambiental, la desigualdad económica, la seguridad alimentaria y la pérdida de biodiversidad. Con los objetivos de aumentar el valor moral y sentimental hacia el mundo natural, transformando la relación que se tiene con la naturaleza y buscando que se respete la tierra (Gilman 1995: 1).

En este punto podemos rescatar ciertos elementos centrales para la comprensión de las ecoaldeas en tanto que fenómeno social:

1) las ecoaldeas han sido una respuesta a una problemática social anterior, basada ésta en la crisis del medio ambiente y/o ante la pérdida de sentido del modelo de desarrollo del capitalismo contemporáneo,

2) la respuesta se basa en superar el momento negativo de la crítica a... de la mera oposición desde la movilización, adoptando la construcción de alternativasviables y factibles en un presente-futuro en proceso de constitución, dejando a un lado las acciones colectivas clásicas de los movimientos sociales del siglo XX,

3) la naturaleza de la respuesta está centrada en un espíritu integral, 
como se lee en Gilman, es decir, es un intento por no dejar afuera ninguna de las dimensiones posibles de la vida social y humana, integrándolas desde la mayor coherencia posible a partir de una visión holística,

4) las ecoaldeashan sido un fenómeno comunitario que impugna la condición y los efectos individualizantes de las formas de vida contemporáneas; el punto de partida es volver a reconstruir los lazos comunes-comunitarios con aquellos que nos hemos distanciado, con los otros que están ahí y se han vuelto invisibles o irrelevantes: la respuesta es colectiva, no individual,

5) han sido y son una praxis y un discurso que pone en igualdad de condiciones lo humano con lo natural o, como algunos han dicho, donde el antropocentrismo le cede el puesto al biocentrismo. La importancia de los ecosistemas y de su equilibrio como condición necesaria para la vida de la especie humana irrumpe en la figura de las ecoaldeas como síntesis de esta necesidad urgente del presente planetario que vivimos. No es que lo humano no sea importante, sino que las vidas de los seres humanos dependen directamente del medio ambiente y de los ecosistemas en los que éstosse encuentran ubicados, y

6) son un retorno o regreso a la tierra en su riqueza y complejidad; movimiento contramigratorio neorrural que va en dirección contraria a lo que ha sido el proceso general de consolidación de lo urbano en los siglos XIX, XX y XXI. Se consolidan en la recuperación de lo rural a partir de la resignificación de la producción agrícola y no agrícola y de los valores articulados a vivencias de una territorialidad construida directamente por los participantes.

Ahora bien, es conveniente entender los alcances y la amplitud de este fenómeno social para hacerse una representación justa de las ecoaldeas. A primera vista, pareciese una reacción minoritaria de sectores poblacionales que han alcanzado un grado de consciencia ecológica profundo y que tienen las condiciones materiales para “dejarlo todo” (muy entrecomillas) y poder disfrutar burguesmente del campo en compañía de sus otros iguales. Esta percepción es realmente errada si tomamos en cuenta los alcances y las dimensiones globales que han conseguido en las últimas décadas.

Lo primero que es necesario afirmar es que las ecoaldeas han tenido un proceso de expansión en todo el mundo ${ }^{3}$, especialmente desde los años noventa, consolidándose como un movimiento global desde respuestas locales ante problemas urgentes medioambientales y sociales. Luego de la Cumbre de la Tierra de Río de Janeiro en el año 1992, en donde se vio la necesidad de impulsar comunidades sostenibles que concretaran el cambio hacia un mundo realmente viable en términos ambientales, sustentables, se produjo la creación de la Global Ecovillage Network (GEN), la Red Global de Ecoaldeas para el año de 1994 (Selba 2014). Al año siguiente se llevó a cabo en Findhorn, Escocia,la primera conferencia mundial sobre "ecoaldeas y comunidades sostenibles” con presencia de más de 400 personas de 40 
países, creándose los tres nodos regionales continentales de la GEN: a) Ecovillage Network of the Americas (ENA) Red de ecoaldeas de las Américas, b) GEN-Europa y c) GEN Asia/Oceanía (Genoa). Sin embargo, la dimensión asociativa del movimiento tiene múltiples ramas en todos los continentes, se puede nombrar como ejemplo la red Sardovaya en Sri Lanka con más de 2.000 ecoaldeas, la Red Ibérica de Ecoaldeas (RIE), la Red Latinoamericana de Ecoaldeas, la Asociación Brasilera de Comunidades Alternativas (Abrasca), la Red Arco Iris de Chile ${ }^{4}$.

Las experiencias más representativas y exitosas en el mundo son, la ya mencionada Findhorn en Escocia, la cual tiene una población aproximada de más de 500 habitantes fijos y una población flotante de 3.000 persona al año (The ecovillage movement today: 2015); comunidad con la más baja huella ecológica del mundo industrializado y premiada por las Naciones Unidas con el premio We the people 50 Communities y con el Best Practice Designation, en los años 1995 y 1998. La ecoaldea de Damanhur en la región del Piamonte italiano es otro de los grandes ejemplos de sustentabilidad del fenómeno asociativo comunitario. Con una población de alrededor de 800 habitantes, ha tenido renombre internacional por la construcción bajo tierra de lo que ellos llamaron los Templos de la humanidad o serie 7 templos creados clandestinamente para la celebración de espiritualidad, abiertos hoy al público. Comunidad igualmente premiada por Naciones Unidas al recibir el Reconocimiento como modelo de sociedad sostenible. En un nivel más amplio, Auroville o ciudad de la aurora, en la India ha representado el proyecto de construcción de la ecociudad más grande del mundo, en donde habitan 1780 personas bajo la idea de una vida comunitaria internacional, más allá de las nacionalidades y los credos políticos, con emprendimientos de carácter ecológico y una idea de armonía con el medio ambiente y formas de acción políticasno-violentas. Así mismo, The Farm, en el Estado de Tennessee, Estados Unidos es otro de los hitos de las ecoaldeas, con cerca de 200 residentes.Con raíces en el movimiento hippie norteamericano de los años setenta, The Farm se ha convertido en una matriz de proyectos ecológicos, educativos, permaculturales y de bioconstrucción. En síntesis, se estima que existen un aproximado de 15.000 iniciativas o experiencias ecoaldeanas en todo el mundo (Ruz 2014 y Litfin 2009).

En Colombia, el fenómeno de las ecoaldeas se ha dado desde comienzos de los años setenta con la puesta en marcha del proyecto Las Gaviotas en los Llanos Orientales, en el Guaviare, el cual desborda la figura de la ecoaldea, llegando a ser calificada como toda un ecopueblo o una ecociudad. La Reserva Integral y Ecoaldea Sasardí quedesde hace 16 años viene siendo otra de las experiencias más visibles. Hoy existe la Red Nacional de Ecoaldeas (Renace) la cual nuclea a unas 12 iniciativas en Colombia y promueve todos los años el encuentro nacional de ecoaldeas, "El llamado de la montaña”, aunque existan otras experiencias que no se reconozcan en esta asociación nacional. Entre las experiencias locales podemos nombrar a Aldeafeliz, en San Francisco de Sales, Cundinamarca; ecoaldea Atlántida en Cajibío, Cauca; El Retoño, en Silvania Cundinamarca; Pachamama en 
Calarcá, Quindío, Anthakarana en Salento, Quindío, Varsana en Cundinamarca y Nashira en Palmira, Valle del Cauca. Como se puede observar a través de toda esta rápida descripción, el fenómeno tiene una dimensión de movimiento global, regional y local organizado, compuesto por diversas redes, tanto en el Norte como en el Sur, tanto en las sociedades más desarrolladas como en el mal llamado Tercer Mundo.

\section{Las ecoaldeas comoobjeto de estudio social}

A pesar de la aparente novedad de las ecoaldeas como fenómeno social, éstas contienen en sí mismas una dimensión epistemológica y ontológica bastante compleja e interesante que implica múltiples dimensiones y problemáticas, las cuales vamos a tratar de abordar en las páginas siguientes. Primero que todo, las ecoaldeas(comunidades intencionales) nos remiten de forma directa al debate clásico dentro de la disciplina sociológica, en cuanto a sus conceptos seminales o ideas fuerza, referidos a la relación tanto dicotómica como complementaria entre comunidad y sociedad. Esto lo podemos ver en la importancia dada a estos conceptos por Ferdinand Tönnies, quien es el primero en darle un estatuto científico al concepto de comunidad (Torres 2013: 35). Para Tönnies,el término de comunidad está significando todo lo relacionado con una forma de existencia cultural basada en lo vital, en la cercanía, en lo natural, en la afectividad y en la amistad; frente a la idea de comunidad, la sociedad es caracterizada como una manera de existencia fundada en el egoísmo, la ambición económica, relaciones basadas en el cálculo del contrato social efectivo, la artificialidad y racionalidad de las relaciones comerciales capitalistas. Así, podemos leer:

Comunidad es la vida en común duradera y auténtica; sociedad es sólo una vida en común pasajera y aparente. Con ello coincide el que la comunidad misma deba ser entendida a modo de organismo vivo, y la sociedad como agregado y artefacto mecánico (Tönnies 1947: 21).

Desde un ejercicio interpretativo, podemos decir que esta relación binaria (comunidad-sociedad) no está planteando una posible filosofía de la historia en donde, aparentemente, deberíamos pasar de formas comunitarias de existencia a formas sociales y en las que habría un marcado sesgo ideológico progresista en la superación de un pasado atrasado y formado por comunidades arcaicas y un nuevo mundo basado en la racionalidad y mecanización de la vida colectiva. Para Tönnies, todo lo contrario, comunidad-sociedad son tipos de formas de vida de las poblaciones, en las que la primera parte del binomio es sinónimo de una mayor riqueza y la segunda parte representa una decadencia o degradación. Aunque históricamente a la comunidad le suceda la sociedad (capitalista), esto no implica que la sucesión signifique un mejoramiento, un progreso.Lo que quiere decir Tönnies es que el ser-en-común se ha ido perdiendo o debilitando en las sociedades capitalistas para dar paso a la artificialidad de las relaciones individualizadas y estructuradas en el cálculo de los intereses, esencialmente separados por sus intereses individuales (1947, p. 65). 
Ahora bien, habría que preguntar hasta qué punto la comunidad es sinónimo efectivamente de naturalidad y autenticidad y la sociedad de artificialidad y mecanicidad. Más adelante retomaremos esta pregunta, para, a la luz de las experiencias mismas ecoaldeanas, responder y poner en entredicho esta premisa. Pero lo que nos importa resaltar ahora es que el debate sobre comunidad y sociedad sigue vigente mediante estas nuevas experiencias comunitarias y nos remite a formas actuales del ser social, no habría que cometer la ingenuidad de pensar que la comunidad, como tipo de existencia social, ha sido superada. En esta medida, las comunidades intencionales como las ecoaldeas serían un repliegue actual en lo comunitario en tanto que respuestas críticas a las consecuencias derivadas (¿funestas? ¿perversas?) de las formas societales del capitalismo vigente. Comunidad y sociedad no son conceptos, por tanto, excluyentes, es decir, la comunidad sigue siendo una realidad actual al interior de las sociedades contemporáneas. Incluso es posible ver, desde este punto de vista, que algunas críticas al fenómeno ecoaldeano se fundamentan ingenuamente en una concepción progresista de la historia en tanto que supuesta superación de etapas o niveles sociales, y en donde el ser-en-común significaría una regresión o anacronismo involutivo en el incesante progreso de las sociedades. Lo que está en cuestión precisamente es la concepción progresiva de la historia a través de estas experiencias comunitarias basadas en el Buen vivir.

Hoy podemos afirmar que la relación binaria comunidad-sociedad puede ser ampliada a individuo-comunidad-sociedad como triada indisoluble que señala formas de ser irreductibles y complementarias de ser. Lo interesante del concepto de comunidad como objeto sociológico es que permite acceder a una crítica a la sociedad actual en la medida en que se ubica epistemológicamente en el límite de lo culturalmente aceptado (la sociedad capitalista como factum incuestionable) para desde ahí señalar un nuevo lugar de enunciación y de construcción de sentido social. Así pues, las ecoaldeas reactualizan la tensión y el debate entre comunidad y sociedad, a la vez que ponen en discusión el telos de la individualidad como resultado de los procesos del capitalismo actual. De igual manera, el concepto de comunidad va a sufrir una de las más interesantes transformaciones al ser ampliado y referirse tanto al mundo humano como al mundo biológico: la comunidad ampliada de lo biótico que incluye al ser humano como un elemento más que debe entrar en equilibrio con todo lo demás.

Un segundo aspecto sociológico que emerge del fenómeno comunitario desde las ecoaldeas es un problema que ha sido ampliamente estudiado por Karl Marx y por gran parte de la tradición marxista crítica y es el de la cuestión del fetichismo de la mercancía.Para Marx este fenómeno ideológico es central para entender el funcionamiento real del sistema capitalista, legitimándose mediante su incesante reproducción. En términos generales, el fetichismo consiste en el proceso por el cual el producto del trabajo del hombre (la mercancía) toma vida propia y existencia independiente, olvidándose,en el trascurso del proceso mercantil, la dimensión cualitativa, esto es, su valor de uso, reduciéndose éste a mero valor de cambio en el mercado. Así: 
El carácter misterioso de la forma mercancía estriba, por tanto, pura y simplemente, en que proyecta ante los hombres el carácter social del trabajo de éstos como si fuese un carácter material de los propios productos de su trabajo, un don natural social de estos objetos y como si, por tanto, la relación social que media entre los productores y el trabajo colectivo de la sociedad fuese una relación social establecida entre los mismos objetos, al margen de sus productores (Marx 1976: 37).

Como podemos apreciar, la fetichización se crea en el proceso de abstracción y de igualacióndel trabajo de los hombres y del tiempo invertido en la producción de las mercancías, donde éstas terminan teniendo un carácter fantasmagórico de autocontrol del movimiento y del valor en el mercado, invisibilizando o dejando en un muy segundo plano "el carácter social de los trabajos privados” (Marx 1976: 41), de los esfuerzos personales invertidos para la elaboración de la mercancía.

El problema de este fenómeno es que, como lo mostraron en el siglo XX Lukács y Benjamin, la fetichización se vuelve reificación o cosificación del sujeto: las relaciones sociales en su aspecto cualitativo se reducen al cálculo cuantitativo de las “cosas inertes” (Margulis 2006: 4). El resultado de la fetichización como reificación es, por lo tanto, la deshumanización de las relaciones humanas y el empobrecimiento general de casi todos los aspectos de la vida social en el capitalismo. La fragmentación de las acciones y la pérdida del sentido de éstas serán unos de los efectos de dicha profundización de la fetichización de la mercancía. Esta lógica de cosificación de la vida llegará incluso al ámbito de la creación y del arte, afectando las estructuras culturales de creación del sentido y de la interpretación de la realidad, aplanando, por decirlo así, el acto creativo en el cálculo mercantil de la ganancia.

Ahora bien, uno de los principales pilares o principios manejados por las ecoaldeas en su movimiento de retorno comunitario al campo es el de la autosuficiencia productiva y el autoabastecimiento alimentario (GEN 2015). La producción local y el consumo responsable como respuestas a la intermediación de los mercados globalizados hacen parte de la propuesta ecoaldeana de romper con la fetichización mercantil en donde existe una gran brecha entre el consumidor final de los productos y el productor. Así, los objetivos fundamentales en este aspecto son: "la reivindicación de nuevos esquemas de producción rural para el sostenimiento, la construcción de nuevas prácticas solidarias y de autoconsumo y la apropiación del territorio que bien podría denominarse como de resistencia rural frente a los modelos urbanos de fabricación y supervivencia” (Cortés, Machetá \& Moreno 2012: 21).

En esta medida, las ecoaldeas atacan los efectos de fetichización y de reificación pues: a) vuelven al consumidor un productor de la mayoría de las mercancíasnecesarias para solventar la mayoría de las necesidades primarias y algunas secundarias, b) el productor-consumidor se reconoce tan- 
to en lo que produce como en aquellos con quienesse lleva acabo la producción, esto es, la comunidad local que retroalimenta el proceso, c) las economías locales generan experiencias de comercio que no están ancladas exclusivamente a la monetarización de los intercambios, por medio de la promoción de ejercicios como el trueque e intercambios de productos con los diferentes habitantes de las regiones donde se ubican, lo cual nos lleva a d) la reconstrucción del valor de uso sobre el valor de cambio, aunque esta última siga existiendo en la mayoría de ecoaldeas. Muchas veces lo que prima es el significado comunitario de lo que produce la comunidad frente a la idea de acumulación de ganancias individuales.

De esta manera podemos afirmar, sin lugar a dudas, que lascomunidades intencionales ecoaldeanas están llevando a cabo prácticas críticas contra los modos fundamentales de la economía capitalista, a través de estrategias centradas en la desfetichización de la mercancía y la decosificación del sujeto o rehumanización de las personas en cuanto a su actividad productiva. Y es precisamente ese cambio de paradigma económico el que pone como primera instancia el bienestar de la comunidad y de las personas y no el de la acumulación individual de ganancias o del desarrollo por el desarrollo mismo. Desfetichizar quiere decir volver a reconstituir el sentido del proceso de producción de las mercancías, potenciando la dimensión de uso socio comunal. Con este proceso se resignifican las relaciones económicas y se traen al primer plano lo comunitario por sobre lo individual, el bienestar común por sobre el interés privado.Así, necesitamos explicitar cuáles son esos principios y prácticas por los que se rige el movimiento global-local de las ecoaldeas y comprender su significado sociopolítico.

\section{Principios y prácticas del movimiento ecoaldeano}

El primer aspecto que hay que tratar es el proceso de constitución a partir de ser un movimiento contramigratorio de recampesinización. Este fenómeno de regreso al campo está acompañado por un cambio de paradigma al echar mano de y al resignificar modos de vida indígenas y campesinos: proponiendo una vida en comunidad, produciendo y consumiendo a nivel local, desde la idea de la agricultura orgánica colectiva y protegiendo la naturaleza. El paradigma o modelo contrario es el de la producción industrializada capitalista que se opone en todos los puntos a aquel: vida individualizada y competitiva, producción nacional y global, consumo de mercancías internacionales y usufructo y explotación infinita de la naturaleza como un recurso material más.Algunos objetivos de este fenómeno de recampesinización son:

- Reducir, reutilizar, reciclar, rearborizar.

- Volver a la tierra (material y espiritualmente).

- Abrazar la tierra para proponer otros modelos de desarrollo: -otras economías

-otras agriculturas 


\author{
-otros hábitats \\ -otra organización ciudadana \\ -otras relaciones humanas (Escobar s/f: 17).
}

Estas comunidades se caracterizan por estar contra la tendencia de "ir a la ciudad" buscando un falso progreso, motivados principalmente por la crisis social y medioambiental del planeta.A la base de la recampesinización está el cuestionarse el modo de vida occidental-capitalista, que en palabras de Escorihuela "encierra una trampa de dependencia a través del consumo" (1999: 1). El trabajo representala enajenación mediante la pérdida del sentido de la producción acelerada, que consume la vida de los individuos y los hace objetos del mercado. A su vez, la ciudad es el símbolo más claro del modelo de vida occidental capitalista y el campo, desde esta representación, está cargado de significados peyorativos que estigmatizan la ruralidad desde todo tipo de negaciones: escasez de posibilidades de desarrollo, no educación, incomunicación, precariedad de servicios, aislamiento etc.

Lo que el modelo de vida occidental capitalista y urbano no percibe es que los costos que acarrea la vida en las ciudades son, como su misma lógica interna lo afirma, de grandes proporciones y en múltiples sentidos: "la pérdida de importantes valores humanos, como la amistad, el deseo de compartir, el trabajo colectivo y desinteresado, el contacto con los hijos" (Escorihuela1999). Detrás de todo esto está la pérdida de sentido de comunidad. Los procesos de individualización y competencia radical han llevado a una especie de sin salida donde ya no es posible el ser con los otros.

\begin{abstract}
Cada vez más gente se siente a disgusto con unas rutinas que no han elegido, con una casa que no es más que un nicho en un enorme bloque de nichos y en cuyo diseño y construcción no han podido participar, con una ciudad que se deshumaniza a cada día con la reducción de las zonas de convivencia y el aumento de los carriles para coches, cada vez más grandes, cada vez más rápidos (Escorihuela 1999: s/p).
\end{abstract}

De alguna manera, la vida en las ciudades del siglo XXI cobra menos sentido. La civilización se ha convertido en la negación de la naturaleza. El regreso a lo rural, la vuelta a la naturaleza, está fundamentada en una revalorización o transfiguración valorativa del binomio ciudad-campo. Éste último es sinónimo de tranquilidad, armonía, posibilidades de un desarrollo diferente o de alternativas a la vida urbana, de reencuentros, de tiempo y del compartir con otros. Como lo dice el manual de Gaia, las ecoaldeas permiten: "contar con un lugar seguro en el que criar a los niños; disponer de más tiempo para la familia y para los amigos y dedicar menos tiempo a trabajos estresantes [...] tener más oportunidades para crear negocios caseros o industrias artesanas [...] para dedicarse a actividades creativas como la música y el teatro" (Gaia, s/f: 33). La ciudad se ha convertido en la representación de la inmovilidad, el estrés, la enfermedad, la contaminación, la inseguridad y la competencia desmedida. La recampesinización es una respuesta, en algún grado anticapitalista, que implica: 
un proceso de transición que va en contra del régimen y los intereses técnico-industriales hegemónicos y se traduce en una 'lucha social' impulsada por el aumento del número de campesinos (a través de influjos exteriores o de 'reconversiones') quienes buscan garantizar una mayor autonomía y sustentabilidad (De Matheus 2013: 3).

Ahora bien, el volver al campo no debe ser entendido como una simple reproducción de los modos de producción tradicionales del pequeño productor de alimentos bajo la estructura familiar clásica. Esta nueva forma de ruralidad implica romper, primero que todo, con el dualismo moderno del campo-ciudad, para llenar de nuevos y plurales sentidos las actividades rurales, no anclándose exclusivamente en la producción de alimentos. Como lo ha afirmado Edelmira Pérez:

Es claro entonces que lo rural ya no es equivalente a lo agrícola, y al mismo tiempo que la llamada tercera revolución agrícola implica que lo agrícola no sea exclusivamente la producción primaria [... . La agricultura comprenderá una amplia serie de usos de la tierra, que va desde producciones agrícolas de uso no alimentario hasta el mantenimiento del medio ambiente (Pérez 2001: 22).

La neorruralidad estaría contemplando una gran variedad de actividades ligadas a la vida rural, más allá de la agricultura, aunque sin desconocer ésta. Desde la experiencia tenida directamente en las ecoaldeas vemos esa pluriactividad desde varias experiencias: el ecoturismo, la protección del medio ambiente y de sus sistemas bióticos, la educación ambiental en contacto directo con la naturaleza, la elaboración de productos artesanales y experiencias espirituales de carácter antropológico religioso como construcción de sentido total del ser humano en el mundo, terapias de sanación corporal y espiritual, etc. (Aldeafeliz, 2015). ${ }^{5}$ Para sintetizar este aspecto, se vuelve al campo para reconstruir el sentido perdido en el proceso de individualización capitalista, el cual es estructurado tanto en la relación con los otros como en la relación con la naturaleza.

Este último aspecto nos remite directamente al principio filosófico que guía gran parte del movimiento y de las iniciativas concretas ecoaldeanas y es el fundamento holístico de su ontología y cosmovisión, lo que lo acerca aún más a la concepción del Buen vivirandino. La realidad es un todo interconectado que debe ser entendido como un organismo vivo. La concepción mecanicista y atomista moderna que fragmenta la realidad en partes con sentido propio cada una se deja atrás para asumir una teoría sistémica en la que la tierra, específicamente, es un organismo energético complejo (Litfin 2009: 129). Así, lo social, lo espiritual y lo ecológico son aspectos que se retroalimentan en dinámicas que encadenan el corto plazo con el largo plazo: las consecuencias de las acciones repercutirán en la dimensión de la cual surgió, afectando a las demás. El ámbito social de los seres humanos está interconectado con el mundo ecológico y las consecuencias del primero se están viendo de forma clara en el segundo; a la vez, 
lo espiritual es una dimensión que refleja y reabsorbe los cambios y contradicciones de los otros dos. La fragmentación de la realidad interconectada ha llevado a la autodestrucción: la separatividad es sinónimo de autodestrucción y muerte.

La concepción holística o cosmovisión holística del movimiento de ecoaldeas se profundiza en cuanto a los modos de vida que deben ser llevados por los participantes. El objetivo es llegar a comprender la interrelación de los elementos que constituyen la realidad y, por tanto, la necesidad de lograr la coherencia y la armonía.

Todos los aspectos de la vida de un individuo, desde su nacimiento hasta su muerte, forman parte de una cadena, cuyos eslabones tienen que estar unidos entre sí, manteniendo una coherencia que permita a cada ser el sentirse y ser parte del mundo en que vive (Ruz 2014: 1).

El sentido profundo del sujeto está configurado por ese "sentirse y ser parte” del mundo, lo que significa superar el individualismo y la fragmentación generada en la vida moderna, sin que esto represente la muerte de la personalidad aplastada por la comunidad. Las ecoaldeas permiten iniciativas personales y privadas económicas y culturales como segunda instancia, luego haber fortalecido proyectos colectivos. Para concretar tal cosmovisión holística se echa mano del concepto de cocreación: "trabajar en comunidad, crear en equipo, valorar más el trabajo en equipo que el trabajo individual. Cultivar una actitud solidaria” (Aldeafeliz, 2015).Se promueveconstantemente la creatividad compartida, la expresión artística, actividades culturales, espacios continuos para rituales y celebraciones, música y danza; con un sentido de apoyo mutuo, unidad y comunidad para mantener la cohesión del grupo.

El principio holístico se profundiza aún más en lo concerniente a la producción agrícola, asumiendo el modelo de la permacultura como guía de producción ecológica, responsable y sustentable. Neologismo derivado etimológicamente de la contracción entre "permanente agricultura” y "permanente cultura”, la permacultura puede ser definida a partir de la idea del diseño de ecosistemas productivos que tienen la estabilidad, la diversidad y la flexibilidadde los ecosistemas naturales (Mollison, 1974), en donde se integra la vivienda, los sistemas de producción de energía, las plantas comestibles, los animales silvestres y domésticos y los recursos hídricos. Así,

La permacultura aprovecha todos los recursos y aúna la mayor cantidad de funciones en cada elemento del paisaje y la mayor cantidad de elementos que sean posibles en cada espacio vertical y horizontal. El exceso o desecho producido por plantas, animales y actividades humanas son utilizados para beneficiar otros elementos del sistema (Young, 2009).

El principio básico de acción de la permacultura es el de "trabajar 
con la naturaleza y no contra ella” (Ruz, 2009). La integración de elementos y funciones es fundamental para lograr esa visión y práctica coherente de los sistemas productivos y así poder llegar al objetivo absolutamente revolucionario de la inexistencia del desperdicio o de la basura. Estos conceptos, como bien lo afirma Litfin (2009: 129), son una creación de la modernidad industrial. "Si los sistemas humanos fueran diseñados de acuerdo a los principios naturales, no habrían desperdicios” (Ibid). Desde diferentes estrategias, la permacultura como diseño consciente, holístico y ecológicamente responsable es desarrollado por la mayoría de ecoaldeas desde: el aprovechamiento de aguas lluvias, los sistemas de riegos naturales, el tratamiento de aguas grises y negras, la producción de compostaje, la producción de energía propia, el reciclaje constante para elaboración de elementos de construcción (bioconstrucción), utilización de corrientes de aire, el uso de abonos orgánicos y el no uso de pesticidas para el control de plagas en la producción de alimentos orgánicos (Ruíz 2012) ${ }^{6}$.

Como se puede colegir de este método de producción responsable, el movimiento de ecoaldeas no está basado en un desprecio por la tecnología ni significa un regreso o involución a formas de vida premodernas, sino que echa mano de las técnicas que sean coherentes con la sustentabilidad ambiental para poder trascender la economía anclada en la dependencia a los combustibles fósiles y así reducir la huella ecológica. El concepto que se trabaja en el movimiento es el de tecnología apropiada, no el de negación de la tecnología (Gaia s/f: 92).

Desde la dimensión política, el movimiento se rige a partir de la crítica implícita a los sistemas de gobierno contemporáneos que delegan la responsabilidad en otros, haciendo insuficiente y lejano el vínculo con las personas que componen la sociedad, aunque muchas veces los ecoaldeanos prefieran no verse como actores políticos. La crisis de los partidos políticos y del sistema de representación democrática, así como la necesidad de enriquecer la dimensión política de las personas han potencializado una forma de comprender la acción concertada y constantemente participativa como una instancia definitiva para responder ante las necesidades urgentes del día a día de estas comunidades. El significado de lo político se retrotrae a la experiencia de la participación directa en la comunidad y para la comunidad, con los objetivos de consolidar un bienestar colectivo o público y de lograr esa recuperación del sentido perdido en el individualismo posesivo. La comunidad, como espacio gestionado colectivamente, compromete un carácter a la vez político, además de social, puesto que conlleva la toma de decisiones colectivas (asamblearias) y la definición de medios y fines para la realización concreta del bien común o del derrotero a seguir como organismo vivo comunitario.

En esta medida, algunas ecoaldeas han tomado la metodología denominada como sociocracia para dinamizar la toma de decisiones bajo un ambiente y una estructura realmente horizontal y participativa. En ésta, se realizan las decisiones de manera consentida, a través de la figura de los círculos o grupos de personas con objetivos específicos, los cuales se 
entrecruzan semi-autónomamente y son asignados enlaces para entrar en contacto con otros círculos, así como facilitadores que son los encargados de dar todos los pasos necesarios para llevar a cabo las asambleas (KanAwen, s/f: 1). Como lo dice el modelo educativo Gaia para la formación de ecoaldeas: "En el consenso como en los ecosistemas vivos, cada individuo gobierna y es gobernado por la comunidad más amplia en una red de relaciones recíprocas” (s/f: 41). La reciprocidad es uno de los valores políticos ecoaldeanos bajo la idea de la responsabilidad de cada uno de los miembros. Para constituirse como comunidad consensual se hacen necesarios los siguientes elementos: "un compromiso informado con el proceso del consenso, disposición para compartir el poder, un objetivo común y una facilitación efectiva, incluyendo el uso de agendas y acuerdos básicos” (Idem.). Esta forma de ejercicio de poder implica un diálogo permanente en un ambiente de confianza de los participantes, donde las razones y la circulación de las ideas desplazan a la figura del voto como instancia de irresponsabilidad o delegación de la responsabilidad para que otros decidan lo conveniente, lo justo. En esta medida, las ecoaldeas realizan formas de autogobierno sociocráticos que implican un ejercicio de poder de todos hacia todos, en los que no es posible ser sujeto pasivo.

La horizontalidad radical del movimiento, se supone, no debe reñir con los liderazgos emanados de los procesos de concertación y de maduración de las comunidades. Esos líderes serían el resultado acumulado de la sabiduría adquirida en la experiencia comunitaria y simplemente deberían ser vistos como facilitadores o "servidores-líderes" de los proyectos y objetivos planteados (Gaia s/f: 42). Hasta qué punto es factible esta horizontalidad o no verticalidad jerárquica será una cuestión que evaluaremos más adelante. Por el momento se hace necesario resaltar la dimensión o perspectiva del Buen vivir que asume el movimiento como parte de sus principios de acción.

\section{El movimiento de ecoaldeas y el Buen Vivir}

Aunque no exista un consenso absoluto de las influencias indígenas en el movimiento de ecoaldeas a nivel global, podemos decir que desde el ámbito latinoamericano y específicamente colombiano hay una fuerte influencia de elementos de los pueblos amerindios en la filosofía comunitaria ecoaldeana. Propuestas como la del Buen Vivir están siendo integradas a los proyectos particulares y hay una gran cantidad de ideas y significados que se articulan en estos espacios ecológicos y culturales. Aunque no es conveniente limitar la influencia únicamente a la dimensión ecológica del movimiento,ésta debe ser tomada de forma transversal ya que su andamiaje es tan amplio que cobija las otras dimensiones y principios, de tal manera que debe ser tomada de manera integral. Para visualizar esta perspectiva se hace necesario analizar el sentido y los principios del Buen Vivir.

El concepto del Buen Vivir es de origen indígena y sus raíces se han venido recuperando en el pensamiento andino. En Ecuador proviene del 
kichwa "Sumak Kawsay", traducido en castellano como "Buen Vivir”, o de Bolivia del Aymara "Suma Qamaña", traducido en castellano como "Vivir Bien” (Farah, 2007), aunque como lo afirma la Coordinadora Andina de Organizaciones Indígenas (ACOI 2010: 13), la traducción más cercana debe entenderse desde la idea de un convivir y un vivir en plenitud, en armonía material y espiritual. El Sumak Kawsay o Suma Qamaña se presenta en oposición a la visión occidental de la "vida buena" que emerge de las ideologías "bíblicas y aristotélicas” (Walsh, 2009, p. 224) y al concepto materialista del "vivir mejor", moderno capitalista. En el caso bíblico por considerar al ser humano como centro y superior a los otros seres vivos; en el caso aristotélico, al considerar que las reflexiones en torno al ser y al valor del hombre dependen de la polis donde es posible la civilización (el desarrollo en sentido occidental) pues fuera de ella solo hay barbarie.

Esta propuesta se orienta a partir de cuatro fundamentos que buscan el "bien común de la humanidad” desde la síntesis elaborada por Houtart:

(i) utilización sostenible y responsable de los recursos naturales, sustentada en las capacidades humanas para construir y sostener una sociedad en la lógica de conservación y renovación de la naturaleza, (ii) privilegio del valor de uso sobre el valor de cambio, para fortalecer vínculos sociales y un consumo apegado a las necesidades, (iii) ampliación de la democracia en todas las relaciones e instituciones sociales, y (iv) multiculturalidad que abra oportunidades a todos los conocimientos que aporten a la ética del bien común (Farah \& Vasapollo 2011:23-24).

Como podemos apreciar, son varios los puntos de articulación entre el movimiento de ecoaldeas y el Buen vivir, los que son además estructurales, como es la cosmovisión holística comunitaria y cósmica: el ser humano no se constituye como tal sin ser parte de una comunidad y de un territorio que da sentido. Sus ejes son la defensa de la vida en todas sus dimensiones, rescatando el sentido ancestral de "Madre Tierra" o de naturaleza sagrada, con una propuesta de complementariedad y de armonía de las personas con la naturaleza y de las personas entre sí.

Además de lo anterior, hay un centro común en lo que respecta a las economías sustentables alejadas del "imperativo mercantil" del puro consumismo planteado por el capitalismo, siendo críticos frente al modelo neoliberal, que obedece a las normas del mercado, que persiste en privatizar lo natural y lo público y que favorece la explotación de los recursos naturales bajo la lógica de la extracción. Para ello se proponen economías distintas que den más valor al uso y la necesidad que al valor de cambio. La adopción de la figura de la minga o trabajo comunitario es otra expresión de la cosmovisión indígena que se desarrolla en el movimiento. Algunas de otras formas económicas alternativas son el trueque, el uso de monedas locales y la ordenación de fondos comunitarios.

En el aspecto cultural, el movimiento de ecoaldeas, por lo menos en 
Colombia, bebe directamente de referentes simbólicos indígenas, Chibchas más exactamente, al asumir espacios de espiritualidad tales como el Cusmuy o casa del sol y la fertilidad masculina para realizar las ceremonias o "rituales de la palabra” donde se encuentran todos los ecoaldeanos para llegar a acuerdos o festejar momentos especiales de la comunidad;en sus ritmos de vida a través de celebraciones del solsticio de verano y de invierno, y otras fiestas; por medio de la adopción de nombres quechuas y chibchas de gran significado e importancia colectiva.

También encontramos toda una amplia gama de actividades relacionadas con prácticas de salud integral de origen indígena como las danzas sagradas de conexión con la tierra, los temazcales, la sanación a través de las plantas sagradas dinamizadas por chamanes y abuelos, etc. (Aleafeliz 2008: 2) El movimiento ecoaldeano colombiano tiene lugares de retroalimentación de sus saberes y de éstos con la cosmovisión indígena en lo que ellos han denominado "El llamado de la montaña”, encuentro anual que invita a grupos indígenas, comunidades campesinas tradicionales, las ecoaldeas de Colombia, innovadores sociales, educadores y empresarios comprometidos socialmente, que hanconseguido intercambiar sus experiencias y nutrirse mutuamente desde sus diferentes saberes. Precisamente, el último llamado de la montaña se realizó en territorio ancestral del pueblo originario guambiano, en el Cauca (El llamado de la montaña 2015), como una muestra más de influencia y articulación con formas ancestrales de concebir ese gran espectro teórico práctico que es el Buen Vivir.

\section{Conclusiones}

El movimiento de ecoaldeas puede ser evaluado desde múltiples aspectos, evitando caer en juicios totalizantes y radicales que no permitirían una justa analítica del fenómeno. En primera instancia, hay que entender que éstas han representado desde hace más de cuarenta años una praxis alternativa al capitalismo desde las dimensiones económica, cultural y política, tal como fue desarrollado en este artículo. El ataque directo mediante acciones positivas al núcleo de las formas capitalistas de la mercantilización o fetichizaciónmercantil y reificación o cosificación humana es un rasgo fundamental en la posible creación de un modelo diferente, más allá del capitalismo. No obstante, las prácticas de desfetichización llevadas a cabo no se muestran como un nuevo modelo post-capitalista o anticapitalista desde el movimiento mismo. Uno de los problemas que hemos detectado es precisamente, en la gran mayoría de casos estudiados, por lo menos en América Latina, es que para llegar a hacer parte de uno de estos proyectos comunitarios se necesita capital, así sea menor, para poder “salirse” del mundo y de la lógica urbana de vida, entrando a ser partícipe de lo comunitario. Es decir, que el pie de apoyo para acceder al mundo ecoaldeano sigue siendo el capitalismo. El modelo otro (alternativo) todavía no existe y esta versión ecoaldeana del Buen vivir sigue siendo bastante tímida y restringida en su impacto global. 
Hemos dicho que el movimiento se configura a partir de ser una respuesta a la crisis ecológica, cultural y económica del mundo contemporáneo, desde acciones positivas y propositivas en el ámbito local. Así mismo, hemos mostrado cómo tiene presencia en todos los continentes, con lo que se afirma su condición de ser global-local a la vez: respuestas locales a problemas globales. Sin embargo, en cuanto al impacto de sus políticas de vida el movimiento es bastante restringido y los problemas globales siguen teniendo la misma dirección catastrófica de insustentabilidad. Aunque estos pequeños laboratorios puedan mostrar caminos diversos en la solución de los problemas, carecen de una actitud confrontativa hacia los gobiernos e instituciones políticas-económicas (Estado y mercado) y, en esa medida, no afectan las estructuras más profundas del sistema contemporáneo. Estamos de acuerdo con Farah y Vasapollo en que: "a pesar de las crisis del capitalismo y sus efectos, no se advierten -a nivel global- fuerzas políticas contestatarias con capacidad de movilización e interpelación” (2011: 11). Las ecoaldeas siguen siendo eso mismo, pequeños e interesantes laboratorios sociales de praxis sustentables, coherentes, responsables éticamente, micropolíticamente participativos y con un sentido de respeto profundo hacia la vida (humana y natural), despreciando, a la vez, toda forma de movilización y confrontación con los actores capitalistas.

El problema de lo micropolítico, de la creación de formas de vida alternativas dadas cotidianamente, sin que haya un intento por la transformación macropolítica de las grandes instituciones de la sociedad,es que se intenta obviar el carácter de dominación y de totalización de los regímenes políticos actuales. En esta medida, las ecoaldeas le dan la espalda a la macropolítica estatal, creando espacios de gobernabilidad propia, de autonomía comunitaria, sin que puedan asegurarse una continuidad de su ejercicio colectivo ante prácticas autoritarias de los Estados o de actores armados ilegales. La vulnerabilidad de los territorios comunitarios es tan o más grande que la que han vivido históricamente las comunidades indígenas en América Latina. Y este no es un problema menor que tendrán que enfrentar las ecoaldeas en un futuro próximo.

Una dimensión que haría falta analizar con mayor cuidado es el rasgo comunitario o de comunalidad ancestral, pues contradiciendo a Tönnies, esa comunidad intencional puede llegar a ser igual de artificial y conflictiva que la sociedad moderna: atravesada por egoísmos, jerarquías, ambiciones, afán de reconocimiento, etc. Esta nueva comunidad, a pesar de todo, es decir, a pesar de toda la naturalidad y holismo que se le quiera adjudicar, sigue estando compuesta por individuos formados en estructuras culturales occidentales (la mayoría de sus miembros son profesionales desencantados de la vida urbana). Valdría la pena responder a la pregunta de hasta qué punto la conflictividad y el individualismo son dinamizadores de lo comunitario, más allá de los principios de armonía profesados por ellos mismos. La comunidad no es garantía inmediata de Buen vivir, sino un lugar y una forma de existencia social que implica esfuerzo, canalización de la conflictividad y lucha del individuo con los modos más profundos de subjetividad inculcados en la matriz capitalista. 
Desde un aspecto aún más contradictorio con respecto a la matriz indígena del Buen vivir, gran parte de las ecoaldeas asumen un lenguaje y unas prácticas que continúan siendo capitalistas para dinamizar la economía interna de las comunidades, ofreciendo experiencias ecoturísticas, de transformación y mejoramiento espiritual de alto costo o de ecoconstrucción para una elite muy selecta de la sociedad, entrando en franca oposición con la lógica del Buen vivir de los pueblos andinos y amazónicos ${ }^{7}$. La alternatividad del movimiento parece flaquear en este aspecto.

Para cerrar este texto es conveniente afirmar que más allá de todas las posibles críticas al movimiento de ecoaldeas que le hagamos, más allá de las contradicciones en las que las comunidades puedan llegar a caer en su intento de sobrevivencia, éstas representan valiosos intentos por crear modos de vida diferentes a los que la matriz consumista del capitalismo contemporáneo nos ha enseñado a seguir, anclados en el necesario ser-encomún vital. Con respecto a la pregunta abierta sobre si es viable un nuevo modelo de sociedad post-capitalista mediante las prácticas concretas de Buen vivir ecoaldeanas, la respuesta estaría más bien enfocada en que éstas son intentos múltiples de reducción de las consecuencias perversas de la sociedad consumista e individualista, y en este proceso puede ser que hallemos poco a poco respuestas más generales y estructurales ante las contradicciones del capitalismo actual. 


\section{Notas}

${ }^{1}$ Este trabajo hace parte de la línea de investigación Análisis interdisciplinario de fenómenos sociales y culturales, del grupo de investigación Pensamiento, Filosofía y Sociedad de la Corporación Universitaria Minuto de Dios (Uniminuto), Bogotá, Colombia.

${ }^{2}$ Es necesario aclarar que, aunque el uso del término estabiliza su significado para los años setenta, desde una generación atrás empezaron a aparecer experiencias comunitarias de regreso al campo con una filosofía de vida distinta a la del capitalismo.

${ }^{3}$ Hay que tener en cuenta que aunque este análisis quiere dar cuenta de la experiencia colombiana, en Europa el movimiento es más antiguo y ha alcanzado una proyección bastante importante tanto en lo cuantitativo y como en lo cualitativo.

${ }^{4}$ Contrariamente a como lo afirma Takis Fotopoulos (2000), se puede ver claramente cómo las ecoaldeas sí constituyen un movimiento de carácter global con amplias ramificaciones continentales, regionales y nacionales, las cuales tienen unos objetivos y principios de acción claros y definidos, a pesar de las diferencias entre experiencias europeas y latinoamericanas o entre éstas y las asiáticas.

${ }^{5}$ Algunas de estas actividades relacionadas con la nueva ruralidad las pudimos comprobar directamente en trabajo de campo realizado entre los meses de mayo y noviembre del año 2014 en las ecoaldeas El Retoño, la casa en el árbol, Silvania, y Aldeafeliz, San Francisco Cundinamarca, Colombia.

${ }^{6}$ Los principios básicos de la permacultura son, según Juan Manuel Madrigal: 1). Ubicación relativa o situar en el lugar correcto. 2). Cada elemento cumple muchas funciones. 3). Cada función es soportada por muchos elementos. 4). Planificación eficiente de energía. 5). Planificación eficiente de recursos biológicos. 6) Reciclaje de energía. 7). Sistemas intensivos a pequeña escala. 8). Aceleración de la sucesión y la evolución. 9). Diversidad. 10). Valoración de los bordes o ecología de la interfase como flujo de energías. Tomado y reconstruido de Madrigal (2010: 3).

${ }^{7}$ El ecoturismo, por ejemplo, es entendido por estos pueblos como parte de la lógica del capital, en donde ellos terminan siendo un apéndice del capital turístico o "mano de obra para el mercado del entretenimiento" (CAOI 2010: 27). 
Polis, Revista Latinoamericana, Volumen 14, No 40, 2015

\section{Bibliografía}

Aldeafeliz (2008), Boletín Aldeafeliz. Recuperado de: http:// www.aldeafeliz.org/descargas/boletin2.pdf

Idem (2015), Página oficial. Recuperado de: http://www.aldeafeliz.org/ index.php?option=com_content\&view=frontpage $\&$ Itemid $=1$

Álvaro, Daniel (marzo de 2010), "Los conceptos de 'comunidad' y ‘sociedad’ ” de Ferdinand Tönnies, en Papeles del CEIC, № 52.

Azkarraga, Joseba, Sloan, Tod, Belloy, Patricio \& Loyola, Aitzol (2012), "Ecolocalismos y resiliencia comunitaria frente a la crisis civilizatoria. Las iniciativas de transición”, en Polis No 33, Polis Revista Latinoamericana, Santiago.

Barney, Harry (2011), Expresión económica solidaria. Un camino hacia el desarrollo humano. Un estudio de caso en la ecoaldea Atlántida. (Vereda Cenegueta, municipio de Cajibío, Cauca, Colombia). Tesis de Maestría en Economía, Universidad Bolivariana de Chile, Santiago.

Bates, Albert (2003), "Ecovillage Roots (and Branches). When, where and how we re-invented this ancient village concept", en Communities Magazine. Disponible en: http://www.gen.ecovillage.org/iservices/publications/ articles/CM117RootsandBranches.pdf

CAOI (2010), Buen vivir/Vivir bien. Filosofía, políticas, estrategias y experiencias regionales andinas, CAOI, Lima.

Cedrssa (2006), Nueva ruralidad. Enfoques y propuestas para América Latina. Cedrssa-Cámara de Diputados, México D.F.

Cortés, Germán Andrés, Machetá, Andrés Mauricio \& Moreno, Luis Eduardo (enero-junio, 2012), "Sustentabilidad y dinámicas organizativas de la ecoaldea "Aldeafeliz" desde una mirada crítico emergente", en Revista Pretil, $\mathrm{N}^{\circ} 26$, pp. 9-22.

De Matheus e Silva, Luis Fernando (2013), “ 'Sembrando nuevos agricultores’: Contraculturas espaciales y recampesinización”, en Revista Polis $\mathrm{N}^{\circ}$ 34, Polis Revista Latinoamericana, Santiago.

De los Ríos, Patricia (1998), "Los movimientos sociales de los años sesentas en Estados Unidos: un legado contradictorio”, en Revista Sociológica, $\mathrm{N}^{\circ} 38$.

Escobar, Federico (s/f), Vámonos pa'l monte. Quince ejemplos de iniciativas de ecoaldeas y reorruralismo en Sudamérica. Recuperado de http:// www.permacultura.cl/boletin/vamonospalmonte.pdf 
Escorihuela, José Luis (s/f), Ecoaldeas y comunidades sostenibles. Recuperado de: http://www.selba.org/UlisesEscritos/EcoaldeasYComuSos.pdf

Idem (1999), ¿Por qué no ecoaldeas? Un espacio amplio para una comunidad difusa. Recuperado de: http://www.selba.org/UlisesEscritos/ PQnoEcoaldeas.pdf

Farah, Ivonne \& Vasapollo, Luciano (Coord.), (2011), Vivir bien. ¿Paradigma no capitalista? Cides-Umsa, La Paz.

Fotopoulos, Takis (julio de 2000), Las limitaciones de las estrategias de estilo de vida: el "movimiento" de ecoaldeas no es el camino hacia una nueva sociedad democrática. Recuperado de: http:// www.democraciainclusiva.org/txt/limit.pdf

Gaia (s/f), Educación para el diseño de ecoaldeas. Recuperado de: http:// www.gaiaeducation.net/docs/EDESpanish.pdf

Gilman, Robert (1995), “¿Por qué ecoaldeas?”, en Ecoaldeas y comunidades sostenibles (modelos para el siglo XXI), Escocia, Fundación Findhorn.

Kanawen (s/f), Información general. Sociocracia. Recuperado de: https:// loomio-attachments.s3.amazonaws.com/uploads/f98f0ddc $4 \mathrm{ffa}$ 205c248570cc080d28bc/Sociocracia\%20informacion\%20general.pdf

Kibbutz Org. (2014), El movimiento kibbutziano. Sembrando esperanza para las generaciones futuras. Recuperado de: http://www.kibbutz.org.il/ tnua/dover/dafdefet_span.pdf

Litfin, Karen (2009), "Reinventing the future. The global ecovillage as holistic knowledge community”, en G. Kütting \& R. Lipschutz (Eds.), Environmental Governance: Power and Knowledge in a local global World, pp. 124-142, Roudledge, New York.

Madrigal, Juan M. (2010), Permacultura: el arte de curar la tierra. Recuperado de: http://cooperma.ourproject.org/wp-content/uploads/2008/05/ la_permacultura.pdf

Margulis, Mario (2006), Ideología, fetichismo de la mercancía y reificación. Recuperado de: http://www.sociales.uba.ar/wp-content/uploads/6-MarioMargulis.pdf

Marx, Karl (1976), El capital. Crítica de la economía política, F.C.E., Bogotá.

Méndez Sastoque, Marlon Javier (2012), “El neorruralismo como práctica configurante de dinámicas sociales alternativas: un estudio de caso”, En Revista Luna Azul, Nº 34. 
Méndez, Marlon, Ramírez, Luz y Alzate, Alejandra (2005), “La práctica de la agricultura urbana como expresión de emergencia de nuevas ruralidades: reflexiones en torno a la evidencia empírica”, en Cuadernos de Desarrollo Rural, (55), 51-70.

Mollison, Bill (1974-1981), Post capitalismo: permacultura y el permacultivo, (Serie de doce videos) [En línea] [Recuperado el 26/11/13] Disponible en: http://www.youtube.com/watch?v=489XPw8tQXQ

Monllor, Neus (2013), "El nuevo paradigma agrosocial, futuro de nuevo campesinado emergente”, en Polis N 34, Polis Revista Latinoamericana, Santiago.

Montoya, Iván \& Dávila, Celia (ene-jun de 2005), “Antecedentes y evolución del sistema de asentamiento y de los Kibbutzim en Israel (1881-1944)”, en Revista Innovar, Vol. 15 N²5 pp. 36-63.

Pereira Salazar, Claudio Antonio (2013), “Participación y acción colectiva en los movimientos globales de ecoaldeas y permacultura”, en Revista Latinoamericana de Psicología, Vol 45, N³, pp. 401-413.

Pérez, Edelmira (2001), Hacia una nueva visión de lo rural, Clacso, Buenos Aires.

Ramiro Avilés, Miguel Ángel (2005), “Paternalismo jurídico y moralismo legal en una sociedad multicultural: el caso de las comunidades intencionales (a propósito de The Village)", en Derechos Fundamentales, Valores y Multiculturalismo, ed. F.J. Ansuategui, Dykinson, Madrid, pp. 111-151.

Rosas-Baños, Mara (2013), "Nueva ruralidad desde dos visiones de progreso rural y sustentabilidad: Economía ambiental y economía ecológica” en Revista Polis $N^{\circ}$ 34, Polis Revista Latinoamericana, Santiago.

Ruz Buenfil, Alberto (2014), Ecoaldeas: alternativas urbanas y rurales, Recuperado de: http://www.absolum.org/eco_aldeas.htm

Selba (s/f), Ecoaldeas y comunidades sostenibles. (Modelos para el siglo $X X I)$, Recuperado de: http://www.selba.org

Torres Carrillo, Alfonso (2013), El retorno a la comunidad. Problemas, debates y desafíos de vivir juntos, Cinde-El Búho, Bogotá.

The ecovillage news org (2015), The ecovillage movement today (2015), Recuperado de: (http://www.ecovillagenews.org/wiki/index.php/ The_Ecovillage_Movement_Today)

Tönnies, Ferdinand (1947), Comunidad y sociedad, Losada, Buenos Aires.

Suárez Rueda, Mariana (2008), Volver al pasado. El boom de las ecoaldeas, 
Recuperado de: http://www.elespectador.com/impreso/articuloimpresovolver-al-pasado

Valdés Kuri, Laura \& Ricalde de Jager, Arnold (Comp.), Ecohabitat. Experiencias rumbo a la sustentabilidad. Semarnat, México D.F.

Vargas, Sandra Bibiana (2009), "Ruralidades emergentes y dinámicas territoriales: nuevas percepciones y medios de vida”, en Revista Eleuthera, Vol 3. (194-205).

Young, Diana (2011), Permacultura o Permacultivo “una ciencia con ética”. Agricultura sustentable y permanente. Bay Area Permaculture. [En línea] [Recuperado el 29/11/13] Disponible en: http://www.mundoalerta.net/ t8902-permacultura-permacultivo

Walsh, Catherine (2009),Interculturalidad, Estado, sociedad. Luchas (de)coloniales de nuestra época, Universidad Andina Simón Bolívar/AbyaYala, Quito.

Recibido: 27.03.2015

Aceptado: 10.04.2015 